\title{
A versatile electronic switch with ncmory
}

\author{
E. C. T. WALKER and D. O. PETTIJOHN \\ Massachusetts Institute of Technology \\ Cambridge, Massachusetts 02139
}

This noise-conditioned switch provides a convenient interface between $S$ response switches and logic circuitry and is adaptable to many experimental situations.

Many of our experiments measure the latency with which a $S$ makes a choice reaction. Usually, we start a stopwatch when a stimulus is presented and stop it when the $S$ reacts, recording the S's response latency and indicating the choice made. The reaction times and choices of up to six Ss are recorded after each trial.

Since we do not wish electrical noise or accidental "responses" by the Ss to operate the stopwatches, we require that our switching devices provide one and only one change of state for each trial. We prefer also that the switches, particularly those which start the stopwatches, provide no audible cues (clicks, etc.) to the $\mathrm{S}$ and that the inconsistent switching times of mechanical and electromechanical switches introduce no extraneous variance into our measures of latency.

The electronic switch described below delivers start and stop pulses of extremely rapid transition to the inputs of electronic stopwatches. Either of two responses by a $\mathrm{S}$ (e.g., yes or no) serves to stop a stopwatch and light an indicator lamp, identifying the response chosen. The switch will not deliver another start or stop pulse until it has been reset by the E. The start, stop, and indicator functions of the switch are modularly organized so that the number of individual switches can be varied as required. The inputs may be wired so that each switch operates individually or so that any combination of start and stop switches operates simultaneously. Since the switch is not mechanical, but electronic, switching times are extremely short $\geqslant 30 \mathrm{nsec}$ ) and consistent. Operation is, of course, electrically and acoustically silent.

The essential logic element in each switch is a J-K flip-flop $1 / 2(74107)$. In the initial state of the flip-flop (after a reset pulse), one output, Q, is held low and the other output, $\bar{Q}$, is held high. A negative-going pulse delivered to the clock input of the flip-flop switches the output at $\mathrm{Q}$ to high and that at $\overline{\mathrm{Q}}$ to low. $\mathrm{Q}$ and $\overline{\mathrm{Q}}$ remain in these new states until they are returned to their initial levels by a pulse delivered to the reset input of the flip-flop. This memory capacity eliminates false switching due to electrical noise or accidental responses. That is, once the flip-flop has been switched, it is immune from further inputs (noise) until it has been reset (see Fig. 1). Using a J-K flip-flop to provide an interface between mechanical switches and logic circuits is a familiar means of coping with electrical noise, and we believe that our switch should prove useful in a variety of experimental situations.

The stopwatches we use also are started or stopped by the negative-going edge of a pulse. The electronic switch provides a start pulse to the stopwatch from the $\bar{Q}$ output of a monostable multivibrator (74121) when triggered by the change of the $\bar{Q}$ output of a flip-flop to low. The pulse width of the "one-shot" (74121) may be set at $\sim 30 \mathrm{nsec}$ or adjusted as desired (up to $40 \mathrm{sec}$ ) by the addition of external timing components. ${ }^{1}$ The $Q$ and $\bar{Q}$ outputs of the "one-shot" provide positive or negative pulses, respectively, in response to a negative-going input at $\mathrm{A}$ or to a positive-going input at $\mathrm{B}$. The diagram (Fig. 1) shows the flip-flop and one-shot connected so as to start the stopwatch with the leading edge of the input pulse. (In fact, the propagation time of each device, $<50$ nsec, will negligibly delay the appearance of this pulse at the stopwatch input.)

Each stop switch contains two flip-flops that provide initial switch conditioning and memory for each choice of response. The $\bar{Q}$ output of each flip-flop is connected to two NAND gates $1 / 2(7400)$ functioning as an AND gate (see Fig. 2). Thus, either choice will provide a pulse

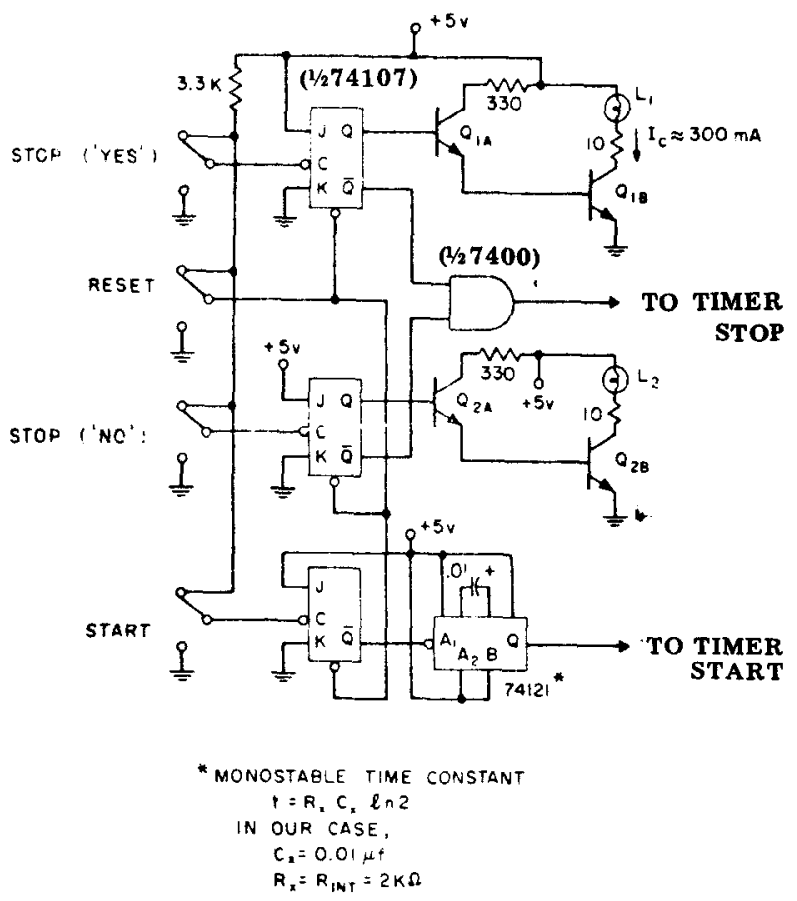

Fig. 1. Circuit diagram.

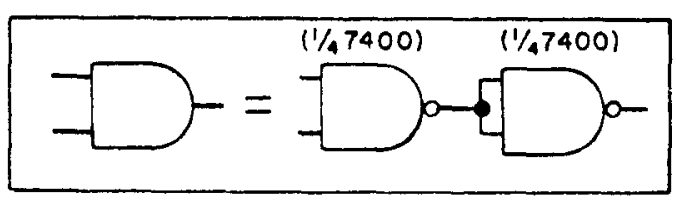

Fig. 2. Two NAND gates functioning as an AND gate. 
Fig. 3. Circuit for power supply of the switch.

\begin{tabular}{|l|l|l|}
\hline \multicolumn{2}{|c|}{$t_{n}$} & $t_{n+1}$ \\
\hline$J$ & $K$ & $Q$ \\
\hline 0 & 0 & $Q_{n}$ \\
0 & 1 & 0 \\
1 & 0 & 1 \\
1 & 1 & $\bar{Q}_{n}$ \\
\hline
\end{tabular}

74107 TRUTH TABLE (APPLICATION OF LO TO RESET FORCES $\overline{\mathbf{Q}} \mathrm{HI}$.)

\begin{tabular}{|c|c|c|c|}
\hline$A_{1}$ & $A_{2}$ & $B$ & $Q$ \\
\hline 1 & 1 & $F$ & INHIBIT \\
0 & $x$ & $Z$ & INHIBIT \\
$x$ & 0 & $Z$ & INHIBIT \\
0 & $x$ & $F$ & $\Omega$ \\
$x$ & 0 & $F$ & $\Omega$ \\
$z$ & $x$ & 1 & $\Omega$ \\
$x$ & $Z$ & 1 & $\Omega$ \\
$F$ & $x$ & 0 & INHIBIT \\
$x$ & 5 & 0 & INHIBIT \\
5 & $x$ & 1 & INHIBIT \\
\hline$x$ & 5 & 1 & INHIBIT \\
7 & $x$ & 0 & INHIBIT \\
$x$ & $z$ & 0 & INHIBIT \\
\hline
\end{tabular}

74121 TRUTH TABLE

Fig. 4. Truth tables.

Table 1

Parts List (All ICs 7400 Series TTL)

\begin{tabular}{ll}
\hline 7400 & Quad 2-Input NAND Gate \\
74107 & Dual J-K Master-Slave Flip Flop \\
74121 & Monostable Multivibrator \\
$\mathrm{Q}_{1 \mathrm{~A}}, \mathrm{Q}_{2 \mathrm{~A}}$ & 2N3904 NPN Hi-Beta Switch \\
$\mathrm{Q}_{1 \mathrm{~B}}, \mathrm{Q}_{2 \mathrm{~B}}$ & 2N2270 NPN, 5 W \\
$\mathrm{L}_{1}, \mathrm{~L}_{2}$ & GE 222 Lamp \\
FWB & 50-V PIV Full-Wave Bridge \\
& (Mallory FW 50 or equivalent) \\
LM 309K & Linear Ic 5-V Regulator \\
\hline
\end{tabular}

to the stop input of the stopwatch. By adding flip-flops and gates, the number of choices can be increased.

The Q output of each stop flip-flop is used to light an indicator lamp powered by a Darlington amplifier $(2 \mathrm{~N} 3904,2 \mathrm{~N} 2270)$ as shown in the circuit diagram. The indicator lamp remains lit until the flip-flop is reset. These lamps could be replaced by transistor-transistor logic (TTL) compatible light-emitting diodes (LEDS). The circuit diagram for the power supply of the switch is shown in Fig. 3. A Silicon General LM309K 5-V regulator was used to guarantee good regulation of $\mathrm{Vcc}$. Properly heat-sunk, this regulator will supply $10 \mathrm{~W}$ of 5-V output. ${ }^{2}$

This switch uses TTL integrated circuits that are cheap, reliable, and readily available. The devices change state when presented with the edge of a pulse. In order to ensure that noise does not produce undesired changes of state, all unused inputs are held high $(+5 \mathrm{~V} \mathrm{dc})$ and the pertinent inputs are held high and switched to low (ground). In our application, the start inputs are pulsed by the external contacts of a projection tachistoscope and the stop inputs are pulsed by three-pole microswitches operated by the Ss. Any "switch" providing the required pulse would serve to operate the switch. Because TTL devices are sensitive to noise, Vcc must be shunted to ground by $1-\mathrm{microF}$ and $.01-\mathrm{microF}$ capacitors at the inputs to each circuit board. In general, noise shunts should be provided at the input terminals of any lines longer than 8 in. ${ }^{1}$

Because the TTL family integrated circuits used in the switch are standard, off-the-shelf items, the cost of the switch is minimal. The power supply shown costs approximately $\$ 10$ and suffices for three start-stop indicator modules. The integrated circuits, transistors, lamps, and miscellaneous materials for each two-choice switch we built cost approximately $\$ 5$, although this cost could vary considerably depending on the components on hand, the number of choices required, and the type of indicators used. The parts list is shown in Table 1 .

\section{NOTES}

1. Motorola TTL Integrated Circuits Data Book (1st ed.), Motorola Inc., 1971

2. Silicon General Technical Bulletin No. 109, February 1971.

(Received for publication June 3,1973; revision received July 10.1973 .) 